\title{
Dinámicas Eco protectora: Sanación y Apache en Nandayuri como sistema de buen vivir
}

\section{Eco-protective dynamics: Healing and Apache in Nandayuri as a good living system}

\author{
Tiziana Rossetti \\ GVC ONG Italia / GVC - Grupo de Voluntarios Civiles \\ ID Orcid: https://orcid.org/0000-0002-9752-2918 \\ rossetti.tiziana@gmail.com
}

\section{Resumen}

El presente artículo reflexiona sobre los conceptos de protagonismo comunitario, dinámicas Eco protectora, Sanación y Apache, investigadas en la Comarca Nandayuri que es parte del territorio del Pueblo Indígena de Monimbó. Como ámbito de estudio, se ha elegido el comunitario indígena, porque el propósito es demostrar que el buen vivir es un modelo de vida y de practica todavía existente en muchas de las culturas indígenas de Nicaragua, así como de América Latina, aunque llamado en diferente manera o simplemente practicado y no conceptualizado. Escuchando la voz de sus habitantes, se intenta interpretar la concepción de vida deseable en Nandayuri, registrado los conocimientos y las prácticas sobre sus recursos biológicos y culturales (patrimonio biocultural), valores y saberes, que en su mayoría están transmitidos y basados en la tradición oral y por lo tanto están en riesgo de perderse y desaparecer.

Palabras Claves: comunitario indígena, Nandayuri, dinámicas comunitaria, protagonistas comunitarios, buen vivir.

\section{Abstract}

This article reflects on the concepts of community protagonism and the dynamics of Eco Protector and of Sanación and Apache investigated in the community in the Comarca Nanadayuri that is part of the territory of the Indigenous People of Monimbó. As a field of study, the indigenous community has been chosen, because the purpose is to demonstrate that living well is a model of life and practice still existing in many of the indigenous cultures of Nicaragua, as well as in Latin America, although called in different way or simply practiced and not conceptualized. Listening to the voice of its population, an attempt is made to interpret the conception of desirable life in Nandayuri, registering knowledge and practices about its biological and cultural resources (biocultural heritage), values and knowledge, which are mostly transmitted and based on the oral tradition and therefore are at risk of getting lost and disappearing.

Keywords: indigenous community, Nandayuri, community dynamics, community protagonists, good living. 


\section{Introducción}

El manuscrito presenta una reflexión sobre los conceptos teóricos aprendidos en el curso de Liderazgo II cruzando la tercera cohorte de la Maestría en Antropología y Liderazgo Social, obteniendo aplicación práctica, en el trabajo de campo con la comunidad indígena de Nandayuri, que es el ámbito territorial de referencia de la propuesta de tesis intitulada "Prácticas cotidianas para alcanzar una vida deseable. Concepción de buen vivir en la comunidad indígena de Nandayuri".

Las principales dinámicas interés de la investigación son la eco- protectora, con el fin de rescatar los conocimientos y las prácticas locales para la preservación de la biodiversidad, así como el análisis de los factores que la están perjudicando, y la de sanación o apache, interpretando el concepto de "estar sano" bajo el enfoque ancestral de la "triple armonía", armonía con sí mismo el entorno y la naturaleza. En el ámbito de estas dinámicas pude identificar los protagonistas comunitarios que con sus prácticas marcan la vida comunitaria, poniéndose al frente a representar los intereses de sus sectores (SIJUs) y de toda la comunidad.

Concluye reflexionando cuales situaciones a nivel local, nacional, latinoamericano y global, favorecen y/o desfavorecen el trabajo de investigación haciendo referencia a la propuesta alternativa del Buen Vivir como proyecto político y de vida avanzada por parte de los pueblos originarios de América Latina y a la perspectiva descolonizadora de la Epistemología del Sur.

\section{Marco metodológico}

El presente artículo es producto de estudio y trabajo de campo realizado desde marzo 2018 hasta abril 2019 en la Comunidad de Nandayuri, Monimbó, cruzando la tercera cohorte de la Maestría en Antropología y Liderazgo Social. Para llevar a cabo la investigación y previo a la realización del trabajo de campo se ha revisado fuentes bibliográficas sobre los autores del pensamiento decolonial, que fundamentan el marco teórico de la tesis y la filosofía del Buen Vivir, para alcanzar la comprensión de la teoría epistemológica que sustenta este paradigma y para identificar las distintas corrientes interpretativas, estableciendo nexus teóricos entre las aportaciones realizadas en los países andino-amazóni- cos y las conceptualizaciones académicas en Nicaragua.

Para empezar a hablar del Buen Vivir, hay que comenzar por precisar que hay Buenos vivires, en plural, no en singular. No hay una sola definición del Buen Vivir completa ni acabada. De hecho, para Eduardo Gudynas (2011), una de las características del Buen Vivir es que es un proyecto en construcción permanente, con un fuerte sentido político y local, contextualmente especifico de cada pueblo, y por ende cada pueblo debe definirlo, sistematizarlo y construirlo, apostando a un cambio de paradigma alternativo al antropocéntrico imperante en los modelos de desarrollo occidental.

Nandayuri es una comunidad perteneciente culturalmente el pueblo indígena Monimbó con quien comparte elementos culturales distintivos, sin embargo, existe elementos particulares a esta comunidad al igual que muchas otras de la zona se ven influenciadas culturalmente por la modernidad y la lógica de consumo que alteran su lógica de vida cotidiana, frente a esto la comunidad genera forman de resistencia propias a su realidad cultural.

Se realizó el trabajo de campo en la comunidad de Nandayuri desde marzo 2018 hasta abril 2019 al fin de conocer las principales características de vida cotidiana de sus pobladores y para aproximar a su realidad cultural particular. El enfoque utilizado para la realización de dicho trabajo fue de corte etnográfico que se caracteriza por ser descriptivo y cualitativo, que permite una reflexión y análisis a partir de la observación del contexto de investigación y desde la percepción de los sujetos sociales ou "actores con intereses en juego" (Ulloa 2010).

Se realizó el trabajo de campo en la comunidad de Nandayuri desde marzo 2018 hasta abril 2019 al fin de conocer las principales características de vida cotidiana de sus pobladores y para aproximar a su realidad cultural particular. El enfoque utilizado para la realización de dicho trabajo fue de corte etnográfico que se caracteriza por ser descriptivo y cualitativo, que permite una reflexión y análisis a partir de la observación del contexto de investigación y desde la percepción de los sujetos sociales ou "actores con intereses en juego" (Ulloa 2010). 
Como sujeto investigador tuve que adoptar una posición crítica hacia mí mismo, problematizar las ideas preconcebidas, estar abierta a lo indeterminado e imprevistos, reconocer y valorizar las distintas subjetividades participantes. Así como explica Paulo Freire, hay que tener la capacitad de dejarse orientar por los sujetos, que tienen la capacidad de enseñarme lo que ando buscando y guiar mi preguntas, para hacer de la investigación no una invasión sino un involucramiento respetando la lógica de los sujetos investigados y activos en la investigación (Freire, 1995 y 2001).

\section{El patrimonio biocultural de Nandayuri, comunidad indígena de referencia territorial.}

La comarca de Nandayuri tiene un área de $2.31 \mathrm{~km} 2$ y está situada en la parte Sur-Oeste del municipio de Masaya, cuyos límites son por el norte con Masaya urbano, al sur con el municipio de Catarina, al este con la comarca el Túnel, y al oeste con comarca Los Nanda. La cabecera comarcal es Nandayuri de Abajo. (Ineter, 2012, p. 67). La localidad presenta dos accesos o caminos que se definen como Nandayuri de Arriba y de Abajo. Su ingreso se hace por el barrio indígena de Monimbó tomando como puntos de referencia la plaza o iglesia de Magdalena en dirección sur-oeste. Nanadayuri es parte del territorio del Pueblo Indígena de Monimbó, descendientes directos de los ancestros Mangues (Chorotegas).

La comarca de Nandayuri tiene un área de $2.31 \mathrm{~km} 2$ y está situada en la parte Sur-Oeste del municipio de Masaya, cuyos límites son por el norte con Masaya urbano, al sur con el municipio de Catarina, al este con la comarca el Túnel, y al oeste con comarca Los Nanda. La cabecera comarcal es Nandayuri de Abajo. (Ineter, 2012, p. 67). La localidad presenta dos accesos o caminos que se definen como Nandayuri de Arriba y de Abajo. Su ingreso se hace por el barrio indígena de Monimbó tomando como puntos de referencia la plaza o iglesia de Magdalena en dirección sur-oeste. Nanadayuri es parte del territorio del Pueblo Indígena de Monimbó, descendientes directos de los ancestros Mangues (Chorotegas).

Estudios arqueológicos y testimonios de los habitantes de la zona evidencian constantes hallazgos de material cultural en superficie (material cerámico y lítico), provocados por actividades de remoción de tierra y diseminados en todas las propiedades que circundan el área de estudio, lo cual que demuestran la presencia de asentamientos prehispánicos. Don Ernesto Escobar Namoyure afirma que "en los caminos que conducen a la laguna observé vasijas pequeñas y grandes, también existen rocas talladas por la gente antigua de la zona", me muestra unos propios hallazgos y asegura "cuando trabajo la tierra se levantan fragmentos de barro y ollitas de todos los tamaños y colores". Asimismo, manifiesta que "esta zona fue saqueada por personas en la década de los '90 y las piezas fueron vendidas a varios extranjeros por unos pesos".

Fuentes etnohistóricas consultadas Niemel, Román, Salgado (1998), Salgado, Román, Gurrero, y Bolaños (2006), cerca del área de estudio demuestran que rasgos cerámicos identificados en la comarca de Nandayuri coinciden con el periodo entre Bagaces final e inicios de Sapoa 700-900 d.C, también teorizan que toda la zona Sur-Oeste de la Laguna de Masaya, se mantuvo una población de considerable tamaño antes de la llegada de los colonizadores, distribuida sobre las partes más planas y próximas a la laguna. La Laguna de Masaya debe haber sido la principal fuente de captación de alimentos y de recursos forestales en su diversidad de flora y fauna. Hoy en día todavía son aprovechados por las comunidades circundantes, a pesar de la creciente contaminación que está degradando y poniendo en peligro la flora y fauna de la Laguna.

La principal actividad económica en Nandayuri es la agricultura de maíz, yuca y frijol, además de especializarse en tener talleres de zapatería, artesanía de barro y en fibra textil, tallado de madera y cuero, productos que son comercializados a los mercados locales y llevado por intermediadores en otros departamentos como al exterior del país, principalmente en Honduras y El Salvador. Como parte de su biodiversidad la zona presenta árboles como: guanacaste blanco, jenízaro, madroño, palmera y pequeños arbustos, además de frutales como naranja, aguacate, mamón, jocote, mango y variedades de plátanos. La fauna se caracteriza por especies de aves diversas, lagartijas, garrobos, conejos y animales domésticos y por temporadas son vistos mono cara blanca y mono congo. 
A partir de la definición de dinámicas como - los diversos conjuntos en los que podemos agrupar las diversidades de prácticas que realizan seres humanos en una comunidad (Ulloa 2010) se ha estudiado las dinámicas Eco protectoras y la de Sanación y Apache entre las once diferenciada en las publicaciones del Prof. Ulloa Forero L. F. "Siete pláticas sobre organizaciones de desarrollo hoy" (Ulloa 2010) y "As dinámicas comunitarias" (Ulloa - De Oliveira 2017).

$\mathrm{Al}$ mismo tiempo se investigan las acciones y prácticas actuadas por la misma comunidad o su entorno, que están afectado y modificando esta relación de reciprocidad entre la comunidad y la naturaleza, reconocida como sujeto portador de derechos, en una relación de cuidado (ireciproco?) por parte de los seres humano, en la cual el antropocentrismo esta hoy fuera de lugar, y la utilización de los bienes naturales comunes como recursos naturales debe ser firmemente cuestionada. (Ulloa De Oliveira 2017).

En las visitas de campo se pudo observar que a pesar de ser Nandayuri un área periurbana contigua al barrio de Monimbó, la mayoría de sus habitantes sigue manteniendo prácticas culturales indígena-campesinas, marcados por elementos de relación, de territorialidad, de identidad y de actividad. Por ejemplo el patio sigue siendo el espacio de crías de animales y la siembra de las plantas medicinales, ornamentales y alimenticias y los alimentos son cocinados con el método tradicional de la leña y el fogón. Las actividades predominantes son la artesanía, la agricultura y pequeñas empresas familiares.

La densidad de población es relativamente baja, los propios habitantes se estiman alrededor de 1000 personas, que se caracterizan por tener prolongada presencia de generaciones en el territorio, estrechas relaciones de parentesco y una fuerte base en las relaciones vecinales. Al mismo tiempo los testimonios hacen entrever los profundos vínculos identitarios que mantienen con su espacio. El territorio y la naturaleza son lugares de referencia geográfica, histórica y espiritual: Josefa García, artesana de 30 años afirma "No podría vivir en otro lugar que no sea Nandayuri. Aquí me crié, aquí tengo mi familia, aquí vivieron mi abuelos. Es un lugar bonito, sano y fresco con mucha naturaleza, aquí no se escucha el ruido de la ciudad."

La Profesora Rosa Del Carmen Dávila cuenta "Nandayuri es un lugar con mucha historia. Desde cuando erábamos niños los ancianos nos contabas las historias de los encantos de la Laguna porque allí, la gente en aquellos tiempos iban hacer rituales para pagar algunas ofrendas. Los abuelitos cuentan historias sobre El cadejo, La llorona, La Cegua, Carreta nagua, el Jinete sin cabeza, la gallina ciega, el perro negro y blanco....Los espantos le salen a las personas agüizoteras, pero ellos siempre llevan un mensaje, advierten si te está portando bien o mal, son señales que le llegan a la gente, más adelante le va a pasar algo o de algo trágico muere esta persona. Como el difunto Centena, mucha veces lo siguió la Mona en la paliza de mango y allí murió de un accidente... Hay lugares a los que le tenemos miedo porque allí salieron y hay temporada en que pueden salir de nuevo. Hay un gran chilamatón donde el hijo de Doña Marta, el tal Mario, le salió dos veces la gallina ciega con los pollito... hay un camino obscuro que baja hacia la laguna donde Don Emilio, pasaba todas la horas en este camino, dormía allí, él decía que no le tenía miedo al diablo hasta cuando le salió el animal de verdad, se fue corriendo y nunca regresó allí. También hay un palo de mamón cerca de mi casa, donde vivía con mi mamá, y ella me contó que una noche un gran perrón vino a dejar a mi papá hasta este palo. Lo perseguía porque mi papa no dejaba de ir de fiesta en fiesta."

El recuento de los protagonistas comunitarios permite reflexionar sobre la importancia de remitirse a la histo- 
ria socio-cultural y a la relación con el medio ambiente (patrimonio bio cultural) del pueblo de Nandayuri, para rescatar de la memoria colectiva e histórica los hechos producidos por los hombres del lugar, que ha sido capaz de reproducir prácticas y conservar saberes del pasado, pero también capaz de recrear o crear nuevos modos de comportamiento. La historia y el patrimonio cultural respaldan la continua producción de presente y futuro de un pueblo, en cuanto la memoria le permite apropiarse de su identidad y trasmitirla en el tiempo, soñar y diseñar su horizonte y es fuente de dignidad y resistencia.

Considerando que cada dinámica de un ámbito está vinculada con otras dinámicas de ese mismo ámbito; unas prácticas con otras prácticas, y los ámbitos entre sí (Ulloa 2010), se pretende integrar al estudio de la dinámica eco-protectora con la dinámica de sanación y apache, porque por la cosmovisión indígena del pueblo de Monimbó, estar sano, significa estar bien, de forma física, moral, mental, social, cultural y económicamente, siendo cuidadoso con su propio cuerpo, la sociedad y el medioambiente.

Esta propia visión se relaciona con las nociones de vida plena y armónica promovidas por la propuesta epistemológica del buen vivir replanteando también el concepto de medicina y de salud. "(Buen Vivir es) la satisfacción de las necesidades, la consecución de una calidad de vida y muerte dignas, el amar y ser amado, y el florecimiento saludable de todos y todas, en paz y armonía con la naturaleza y la prolongación indefinida de las culturas humanas.(...) tener tiempo libre para la contemplación y la emancipación, y que las libertades, oportunidades, capacidades y potencialidades reales de los individuos se amplíen y florezcan de modo que permitan lograr simultáneamente aquello que la sociedad, los territorios, las diversas identidades colectivas y cada uno -visto como un ser humano universal y particular a la vez- valora como objetivo de vida deseable (tanto material como subjetivamente, y sin producir ningún tipo de dominación a un otro" (Ramírez, 2008).

Este modelo tiene una visión holística del concepto de salud y vida saludable, que son conceptos complejo y complementario, presupone y produce un modelo de ayuda reciproco en la comunidad y el intercambio dinámico entre la naturaleza, los seres humanos y la colectividad en una relación de "triple armonía".

\section{Protagonistas comunitarios y Sectores con Intereses en Juego (SIJUs) en Nandayuri.}

Con relación a la dinámica eco protectora se pudo identificar que el sector con mayor interés en juego son los agricultores y los productores artesanales porque son las principales actividades para satisfacer las necesidades básicas, asegurando la sustentabilidad del uso de los recursos naturales y representan también un rasgo fuerte de su identidad. Sin embargo, hoy en día, las actividades tradicionales y los recursos naturales están siendo afectados por intereses de actores externos y por ciertas características del modelo de desarrollo capitalista.

Entre los agricultores se encontró a Don Arsenio Escobar Namoyure, un agricultor de 42 años, padre familia, nieto de unos de los fundadores de las comunidad, que aún no se reconoce ni es reconocido como un líder, en el sentido de poder de representación y toma de decisiones, Don Escobar es una persona reservada pero es muy admirada y respetada por parte de la comunidad, por su acciones de cuidado hacia la naturaleza y por ser un ejemplo en este sector.

Entre los agricultores se encontró a Don Arsenio Escobar Namoyure, un agricultor de 42 años , padre familia, nieto de unos de los fundadores de las comunidad, que aún no se reconoce ni es reconocido como un líder, en el sentido de poder de representación y toma de decisiones, Don Escobar es una persona reservada pero es muy admirada y respetada por parte de la comunidad, por su acciones de cuidado hacia la naturaleza y por ser un ejemplo en este sector.

En la dinámica de sanación y apache se pudo identificar como protagonista líder y promotor del estatus quo, la Señora Simona Castro, anciana de la comunidad, que se dedica a cultivar planta medicinal, preparar "remedios" y muchas personas de la comunidad acuden donde ella cuando tiene problemas físicos y emocionales. En su testimonio ella cuenta "No soy médico, y las personas lo saben, a veces vienen a mi casa con dolores en el cuerpo, con alguna enfermedad, para que le deje un remedio. Yo le digo que me cuenten sus problemas y me doy cuenta que en realidad lo que tienen es un 
dolor en el espíritu... Nandayuri es pequeña, todo nos conocemos y lo bueno es que las personas son muy solidarias. Cuando hay un enfermo o se muere un familiar, todos apoyan a la familia, la visitan, le llevan comida, no la dejan solo y este ayuda que la persona se recuperen o a los familiares a recuperarse del luto. Siempre les digo al joven de aquí que no pierdan este amor hacia el otro, que no sean indiferentes hacia el dolor de otra persona, porque si no mantenemos unidos podemos ayudarnos y sanar el uno hacia el otro."

\section{Valoración del Entorno (local, nacional, lati- noamericano, mundial) para el estudio.}

La propuesta del trabajo de maestría hace referencia teórica a propuestas latinoamericana de la epistemología del sur, como alternativa al conocimiento eurocéntrico dominante y que trata de recuperar los conocimientos y prácticas de los grupos sociales que, a causa del capitalismo colonial y los procesos coloniales, fueron históricamente y sociológicamente excluidos.

La epistemología del sur significa el reclamo de nuevos procesos de producción y de valoración de conocimientos válidos, científicos y no científicos y de nuevas relaciones entre diferentes tipos de conocimiento a partir de las prácticas de las clases y grupos sociales que han sufrido de manera sistemática, las injustas desigualdades y las discriminaciones causadas por el capitalismo y el colonialismo. Un sur anticolonial (De Sousa Santos, 2010).

Participar de la producción y de la difusión del conocimiento ha sido la demanda avanzada por muchos pueblos indígenas en América Latina y que radica en una crítica ontológica a lo que el pensamiento crítico latinoamericano ha calificado como "colonialidad del saber" y que como alternativa propone el "dialogo de saberes" o la "ecología de saberes" de Santos. Desde el mundo indígena latinoamericano ha surgido una alternativa de coexistencia con la naturaleza y entre los humanos que se llama "el buen vivir", concepto que junto a lo derecho de la Madre Tierra (o Pachamama) ha sido introducido en las constituciones de Bolivia (2009) y Ecuador (2008).

Estos nuevos conceptos son entendidos como expresión de una crítica profunda a las nociones tradicionales de progreso, desarrollo y bienestar en estos dos países y de sus pueblos, forman parte de un ciclo reciente de transformaciones plurinacionales del constitucionalismo latinoamericano que reconocen la naturaleza multicultural de las sociedades y de los pueblos indígenas como colectividades y nacionalidades distintas, no solo a nivel cultural.
La Constitución Política de Nicaragua (de enero de 1987) establece un régimen de autonomía regional multiétnicas para el ejercicio de los derechos colectivos de los pueblos indígenas (miskitos, sumos y ramas) y comunidades étnicas (creoles, garífunas y mestizos) de la Costa Caribe, definiendo como ámbito territorial dos regiones: la Región Autónoma Costa Caribe Norte (RACCN), y la Región Autónoma Costa Caribe Sur (RACCS), que constituyen en conjunto aproximadamente la mitad del territorio nacional, con una población indígena muy reducida demográficamente. Esto último muestra que lo decisivo en la búsqueda de soluciones a las exigencias de autodeterminación y autonomía de los pueblos indígenas es eminentemente político y no demográfico. Actualmente queda en la espera de aprobación la propuesta de Ley de autonomía de los pueblos indígenas del pacifico, centro y norte, presentada en 2012 a la Asamblea Nacional por parte de la Red de Pueblos Indígenas del Pacifico Centro y Norte de Nicaragua.

En el marco jurídico internacional de protección de los derechos de los pueblos originarios el Convenio 169 de la OIT y la Declaración sobre los Derechos de los Pueblos Indígenas de la ONU, refuerzan el reconocimiento constitucional de la configuración multiétnica y pluri-cultural de sus poblaciones, superado el esquema del Estado-nación, donde la autonomía dentro del Estado puede configurarse como estrategia de afirmación del derecho a la libre determinación de los pueblos indígenas. Sin embargo, se considera desde esta reflexión del estudio que todavía los organismos internacionales, como la ONU, permanecen en su visión eurocéntrica de desarrollo y no han sabido escuchar e integrar en sus planes de acción los planteamientos que vienen desde las instancias indígenas. Por ejemplo temas como el buen vivir, la libre determinación, consentimiento libre, previo e informado no son abordados y tomado en consideración en la Agenda 2030, que contiene los Objetivos de Desarrollo Sostenible (ODS).

Otro factor que considero una fuerte amenaza para la concreta realización del proyecto político y de vida propuesto por "el buen vivir" es la avanzada de sistemas políticos, ampliamente amenazantes de los derechos indígenas y el irrespeto a las libertades, que generen altos niveles de desigualdad social, económica y de exclusión de la vida política de los pueblos originarios, hasta el real riesgo de su extinción, que puede conseguir a las políticas ambientales, económicas y sociales como el que promueve el actual gobierno de Bolsonaro en Brasil. A nivel local la actual crisis política y social que vivió el país afectó inevitablemente el trabajo de maestría, 
incluso podría no permitir el avance del mismo. La comunidad de Nandayuri se encuentra en el barrio de Monimbó, lugar que fue triste escenario de enfrentamientos violentos que dejaron varios muertos y un entorno polarizado. La población local aún tiene rezagos de miedo e inseguridad, podría eso afectar la investigación directamente, ya que al ser una persona externa (investigador) de la localidad y además el nivel de polarización, podría crear malos entendidos en la comunidad con los comunitarios y autoridades locales. Por esa razón, la actividad de campo está parcialmente suspendida.

\section{¿Cuáles preguntas auténticas me quedan aún abiertas?}

Investigando la cosmovisión y los sistemas de valores y saberes como componentes dinámicos de la comunidad, se procura entender y conocer la concepción de vida deseable propia del pueblo indígena de Monimbó que vive en Nandayuri e identificar con ellos cuáles son las necesidades, conocimientos y experiencias, que la comunidad solicita para alcanzarla.

Por este fin, ¿Sería útil, como producto final de la investigación, diseñar con la comunidad un proyecto o estrategia alternativa de sustentabilidad para la comunidad?

También reflexionar sobre ¿Como la cooperación internacional debería actuar para incluir la visión y saberes de los pueblos indígenas en la construcción de "planes de desarrollo" que sean realmente endógeno (no impuesto desde a fuera), representando la real aspiraciones y deseos de las comunidades locales y por lo tanto alcanzarían la auspiciada participación, apropiación y, sobre todo, sostenibilidad?

\section{Conclusión}

Los pueblos indígenas chorotegas del Centro y Norte de Nicaragua, tras cinco siglos de aculturación, han sido despojados de su religión, de su lengua, de su cosmovisión, de sus formas de producción y de sus formas de organización y de parte o de todas tierras comunales.

Monimbó cuenta con un título real de propiedad de 7 caballerías antiguas, que fue otorgado por el Reino de España representado por la Capitanía General de Guatemala el 16 de Marzo de 1.714. Este título se encuentra desaparecido a consecuencia de los conflictos bélicos y persecución a los mismos documentos. Los Estatutos de la Comunidad Indígena de Masaya fueron aprobados por Acuerdo ejecutivo del 14 de enero de 1915, siendo sometidos a revisión en el año 2012 para su adecuación a la legislación contemporánea, como es el caso de la Constitución de la República de Nicaragua, el Convenio
No 169 de la OIT y la Declaración de las Naciones Unidas sobre los Derechos de los Pueblos Indígenas.

A finales del siglo XIX el territorio colectivo de Monimbó fue individualizado por el Estado re- partiéndose en grandes fincas a terratenientes y pequeñas parcelas a familias indígenas y en los años 80 del siglo pasado parte de sus tierras fueron distribuidas a cooperativas de producción. Actualmente la propiedad colectiva se limita a cuatro cementerios indígenas y una instalación para actividades comunitarias, que son administrados por el Concejo de Ancianos de Monimbó.

La pérdida del control del territorio no ha impedido que el pueblo indígena de Monimbó mantenga su continuidad histórica y cultural, con su patrimonio material e inmaterial que es la principal fuente de mantenimiento de su identidad ancestral incuestionable. Como bien lo señala Javier García Bresó, se trata de un caso de identidad étnica especial que ha sido capaz de sobrevivir al paso del tiempo y a los profundos cambios producidos a través de la historia, en la cual en muchas ocasiones fue dominada, marginalizada y humillada.

La comarca de Nandayuri, que pertenece a este territorio, es una población periurbana de tradición indígena-campesina, cuya identidad indígena es fruto del mecanismo de resistencia que Bresó denomina, siguiendo las ideas de Vogt, "encapsulación", es decir, "el proceso por el que nuevos elementos impuestos desde afuera son conceptual y estructuralmente incorporados a patrones existentes de comportamiento social y ritual". Tanto es así que, hoy en día, su identidad se fundamenta precisamente en el hecho de haber mantenido algunas tradiciones de clara influencia colonial, con un apego especial y que ha construido la territorialidad a partir de sus tradiciones y organizaciones propias, como las Alcaldías de Vara, Concejo de Ancianos, la Cofradía y Junta Administrativa.

Sin embargo, los proceso de aculturación se manifiestan en el desconocimiento de la lengua originaria, en la normalización occidental de la educación escolar, en la politización partidaria de los liderazgos comunitarios, en el creciente peso en los ingresos monetarios derivados de la comercialización de los recursos naturales y productos de artesanado, en el trabajo asalariado como temporeros y en la dependencia de la actuación de la municipalidad y del gobierno para mejorar sus servicios básicos (educación, viviendas, caminos, fuentes, electricidad, protección del medioambiente).

No obstante, el fuerte vínculo que los pobladores de Nandayuri tienen con su estructura económica tradicional de producción agrícola y artesanal, con todos sus aspectos relacionados, cuales, ritos, festividades, alimentación, así como con la gran cantidad de leyendas, cuentos, mitos e historias, que se han ido transmitiendo de generación en generación, hace que aun conserve una forma de vida de origen ancestral. Se puede afirmar 
que las comunidades tienen una concepción propia del "buen vivir" que está basada en la vida en "triple armonía", armonía con sí mismo, con la comunidad y con la naturaleza.

De esto elemento de vida en armonía y equilibrio, los monimboseños de Nandayuri atribuyen particular importancia al concepto de "salud", personal y comunitaria, replanteándolo de acuerdo a esta idea de buen vivir. Las enfermedades se conciben bajo una doble perspectiva en la cual por un lado son algo natural y por otro son algo relacionado con la sociedad, la persona puede enfermarse por su mala conducta y por desestabilizar la armonía la convivencia armoniosa con el entorno social y ambiental. En Nandayuri se encuentran diferentes especialistas: parteras, sobadores, curanderos, curanderos de mordeduras de serpientes y ancianos, a esta persona se le atribuye un "don" que reciben del Gran Padre, es un "don" de conocimiento y entendimiento. Todos los especialistas indígenas coinciden en afirmar que la sanación de las personas,

\section{Bibliografía}

Agenda 2030 para el Desarrollo Sostenible. UN. https://www.un.org/sustainabledevelopment/ es/2015/09/la-asamblea-general-adopta-la-agenda-2030-para-el-desarrollo-sostenible/

Bresó, García Javier. (1992) Identidad y Cultura en Nicaragua: estudio antropológico de Monimbó. Tesis Universidad Complutense. Ediciones de la Universidad de Castilla-La Mancha, D.L.

González Pérez, Miguel. (1997) Gobiernos pluriétnicos: La constitución de las regiones autónomas en Nicaragua. Estudio sobre el Estado nacional y el proceso de autonomía regional en la Costa Atlántica-Caribe. URACCAN/ Plaza y Valdés. México.

Niemel, K, Román, M, Salgado, S. (1998). Las Secuencias Cerámicas de los Periodos Sapoa (800-350 d.c) y Ometepe (1350-1522 d.c) en el Pacífico Sur de Nicaragua. En XI Simposio de investigaciones Arqueológicas en Guatemala, 1977 (editado por J.P. Laporte y H, Escobedo). familia y la comunidad se sustenta en la fe del enfermo para la búsqueda del equilibrio espiritual, mental, físico, de comportamiento y de cuidado a la Madre Tierra.

Finalmente, tentando de contestar a las preguntas que quedan abierta, se considera que los proyectos y programas, que tienen como objetivo impulsar la economía local en las comunidades, deben estar estratégicamente vinculados a los conocimientos empíricos, las técnicas, los recursos, las prácticas sociales y culturales locales, para apostar a procesos de desarrollo endógeno, que respeten la base cultural e identidad territorial y expresen las proyecciones, visiones y propuestas locales de desarrollo. La diversidad cultural no debe ser un obstáculo para aspirar al progreso, al contrario, debe ser la base de un rico proceso histórico de interculturalidad permanente y complementariedad en la diversidad, a partir del cual diseñar planes y estrategias con objetivos comunes por el "buen vivir" y el desarrollo humano sostenible.

Santos, B.D. (2010) Refundación del estado en América Latina: Perspectivas desde una epistemología del sur. México: Siglo XXI Editores

Ulloa, L. F. (2010). Dinámicas Comunitarias y unos Parches. Bogotá: S/E.

Ulloa Forero, L. F. (2018). Dinámicas Comunitarias...y unos parches. Bogotá: S/E.

Ulloa Forero, L. F., \& Buriti, C. d. (2017). As Dinâmicas Comunitárias: um olhar para as comunidades desde o que as pessoas fazem na sua vida cotidiana. Campina Grande, Paraiba, Brasil: Instituto Nacional do Semiárido, INSA.

Salgado, S, Román, M, Gurrero, E y, Bolaños, A. (2006). Comparación de las Aldeas Nucleadas de Bagaces (300-800 d.C) del Pacífico de Nicaragua.

RAAN map, http://www.aurorabeachfront.com/ nicaragua/maps/m_raan.gif

Sole, Josep Maria, Arasa Raul et al. "Assessment of Climate Change in Nicaragua, Earth and Environmental Journal, $\mathrm{Vol} \wedge$ number 3, July 2016, Pages 445-474, http://www.scirp.org/journal/PaperInformation.aspx?paper ID=68368, Accessed 24.10.2017 
URACCAN, Wikipedia, accessed 16.10.2017

https://en.wikipedia.org/wiki/Demographics_of_Nicaragua\#Indigenous_population

https://en.wikipedia.org/wiki/University_of_the_Autonomous_Regions_of_the_Nicaraguan_Car-
ibbean_Coast accessed 17.10.2017

Von Houwald, Gotz, Mayangna, Edited in German for the Ethnology Museum in Hamburg, Vol. XIX/1990/, Translation into Spanish Edgard Arturo Castro Frenzel.

\section{Tiziana Rossetti}

Maestrante de la Maestría en Antropología y Liderazgo Social tercera cohorte. Formación principal en Ciencias de la comunicación escrita e hipertextual, de origen italiano. Ha trabajo con la cooperación internacional de Italia, en varios países de Latinoamérica, entre ellos Honduras, Ecuador, Colombia, Brasil, en temas de derecho de la mujer y la niñez, desarrollo comunitario entre otros. Actualmente trabaja y reside en Managua. Desempeñándose en distintos programas de voluntariados internacionales. 